\title{
Hospital Resilience: A Recent Concept in Disaster Preparedness
}

\author{
Boeriu Cristian*
}

University of Medicine and Pharmacy Tîrgu Mureș, Department of Emergency Medicine, Tîrgu Mureș, Romania

Planning for a disaster must anticipate how demands imposed by a disaster equate with the capacity of the available facilities. Resources must be organized before an event occurs so that they are best prepared in every way to treat as many victims as possible. The actual number of victims is less relevant than the extent receiving facility can be adjusted to meet the appropriate requirements of victims. Multiple casualty incidents (MCIs) are defined as a large number of casualties generated over a short period that are appropriately managed with existing or extended resources. Mass casualty events (MCEs), in contrast, are major medical disasters that erode organized community support mechanisms and result in casualty numbers which overwhelm resources [1].

Due to the increased frequency and impact of disasters, including natural disasters, pandemics and terrorism, the concept of disaster resilience is accepted as being of increasing importance.

The notion of resilience can be defined as the capacity to adapt to unexpected challenges and the flexibility to revert to normality. Additionally, the issues learned from the experience should be incorporated into protocols which would allow for better preparedness for future challenges $[2,3]$.

Another definition, linked to disaster management, describes resilience as the capability of a medical facility to withstand the event, while being able to maintain and expand their medical capacity and to respond to sudden and significant increases in patient demand $[4,5]$.

Hospital disaster resilience refers to a hospital's ability to withstand, absorb, and respond to disasters while maintaining critical functions, and then to recover to its original state or adapt to a new one. Understanding the status of a hospital's disaster resilience is the first step in planning to enhance effective emergency response services [6].

Healthcare resilience, especially hospital resilience, is essential as it provides "lifeline" services for mini- mising the impact of disasters on the community and achieving higher community resilience $[2,3]$.

The role of hospitals in response to disasters has significantly increased over the last twenty years due to the concomitant escalation in numbers of large-scale emergencies and natural, technological or terrorist-related disasters. Hospital design and preparedness must be sufficient to deal with and recover from all types of emergencies and disasters [7].

Resilience can be achieved through the "PPRR" management strategy of prevention and mitigation $(\mathrm{P})$, preparation and planning $(\mathrm{P})$, response and relief $(\mathrm{R})$, and recovery $(\mathrm{R})$ [8].

The strategies should be comprehensive, including structural components (e.g. infrastructural safety), non-structural components (e.g. staff capability), emergency medical functions (e.g. critical care, on-site rescue, and surge capacity), and disaster management mechanisms (e.g. plans, crisis communication, and cooperation) $[9,10]$.

All these components can influence or determine the performance of hospitals following a disaster and ultimately influence a community's health outcomes [3].

Given the critical role of hospitals, the model of "safe and resilient hospitals" was promoted as a key component of disaster risk reduction planning in the healthcare sector during the 2005 World Conference on Disaster Reduction. This conference endorsed policies that would ensure "that all new hospitals are built with a level of resilience that strengthens their capacity to remain functional in disaster situations" $[11,12]$.

Albanese et al. showed that the concept of „safe and resilient hospitals" hospitals must encompass and address infrastructure and cross-cutting themes of hospital disaster preparedness including institutional capacity building, education and training, project implementation, facilitating local and regional cooperation, information sharing, networking and knowledge

Correspondence to: Boeriu Cristian, University of Medicine and Pharmacy Tîrgu Mureș, Department of Emergency Medicine, No.38, Gheorghe Marinescu St. 540139 Tîrgu Mureș, Romania. 
management, and the provision of subject matter expertise. Safe and resilient hospitals represent facilities where urgent medical care can be dispensed in an environment which is functioning at full capacity or can operate as a sufficiency-of-care facility. These healthcare units should be capable of providing the reassurance and medical leadership needed by the general public in times of crisis and develop structured relationships that establish an interface among local and regional entities involved in community-wide disaster response. The establishment of "safe and resilient" hospitals is needed to validate the hospitals' importance as a community asset and maximize their integration within a community-wide disaster response [13].

There is little consensus regarding a framework for evaluating hospital resilience, and this has led to confusion in disaster health management research, making it difficult either to evaluate hospitals comprehensively or to identify their prioritized actions for confronting future disasters. Zhong et al. developed a comprehensive framework which integrates key indicators of hospital disaster resilience. The framework provides a starting point for broad agreement regarding the key components of hospital resilience. According to this framework the most critical indicators of hospital disaster resilience are related to emergency critical care capabilities, such as on-site rescue, referral of patients with complex needs, emergency medical treatment, surge capacity, hospital mass casualty triage, and the factors that guarantee it: disaster committee, disaster plan, stockpile and management of medications. Hospital resilience can also be improved through putting in place a wide variety of flexible plans, such as cooperation with other facilities, strategies to prioritize and maintain critical care functions, and strategies to surge emergency resources, medications, and staff [14].

A conceptual understanding of hospital resilience is essential to provide an intellectual basis for an integrated approach to system development. Developing the concept of "hospital resilience" will provide a starting point for agreement about what it comprises and how to measure it. The new concept links these key components with an achievable goal to improve hospital pre-event robustness as well as rapidity to recover and adapt for disasters [15].

Further consensus on key measures of hospital resilience would improve the consistency of a hospital's emergency practices and position it with an improved ability to cope with disasters of all kinds.

\section{CONFLICT OF INTEREST}

None to declare

\section{REFERENCES}

1. Roccaforte JD, Cushman JG. Disaster Preparedness, Triage, and Surge Capacity for Hospital Definitive Care Areas: Optimizing Outcomes when Demands Exceed Resources. Anesthesiol Clin. 2007;25:161-77.

2. Braun $\mathrm{Bl}$, Wineman NV, Finn NL. Integrating hospitals into community emergency preparedness planning. Ann Intern Med. 2006;144:799-811.

3. Paturas JL, Smith D, Smith S. Collective response to public health emergencies and large-scale disasters: Putting hospitals at the core of community resilience. J Bus Contin Emer Plan. 2010;4:286-95.

4. Bruneau M, Chang SE, Eguchi RT et al. A framework to quantitatively assess and enhance the seismic resilience of communities. Earthq Spectra. 2003;19(4):733-52.

5. Cimellaro GP, Reinhorn AM, Bruneau M. Seismic resilience of a hospital system. Struct Infrastruct Eng. 2010;6(1-2):127-44.

6. Zhong $\mathrm{S}, \mathrm{Hou} \mathrm{XY}, \mathrm{Clark} \mathrm{M}$ et al. Disaster resilience in tertiary hospitals: a cross-sectional survey in Shandong Province, China. BMC Health Serv Res. 2014;14:135.

7. Sauer LM, McCarthy ML, Knebel A, Brewster P. Major Influences on Hospital Emergency Management and Disaster Preparedness. Disaster Med Public Health Preparedness. 2009;3(Suppl 1):68-73.

8. Rogers P. Development of resilient Australia: enhancing the PPRR approach with anticipation, assessment and registration of risks. Aust J Emerg Manag. 2011;26:54-59.

9. Veterans Health Administration. Emergency management program guidebook. Washington, DC: VHA Center for Engineering \& Occupational Safety and Health (CEOSH), 2011.

10. Zhong S, Clark M, Hou X-Y, et al. Validation of a framework for measuring hospital disaster resilience using factor analysis. Int J Environ Res Public Health. 2014;11:6335-53.

11. International Strategy for Disaster Reduction. Hyogo framework for action 2005-2015. Building the resilience of nations and communities to disasters. In: World Conference on Disaster Reduction: Hyogo, Kobe. 2005, pp.18-22.

12. Pan American Health Organization. Area on emergency preparedness and disaster relief. Regional Office of the World Health Organization. Annual Report 2005.

13. Albanese J, Birnbaum M, Cannon C et al. Fostering disaster resilient communities across the globe through the incorporation of safe and resilient hospitals for communityintegrated disaster responses. Prehosp Disaster Med. 2008;23(5):385-90.

14. Zhong S, Clark M, Zang YL, Fitzgerald G. Development of key indicators of hospital resilience: a modified Delphi study. J Health Serv Res Policy. 2015;20(2):74-82.

15. Zhong S, Clark M, Hou XY, Zang YL, Fitzgerald G. Development of hospital disaster resilience: conceptual framework and potential measurement. Emerg Med J. 2014;31(11):930-8. 